\title{
Editorial
}

\section{International Task Force on Benzodiazepines}

\author{
Richard Balon $^{\mathrm{a}}$ Guy Chouinard $^{\mathrm{b}}$ Fiammetta Cosci $^{\mathrm{c}}$ Steven L. Dubovsky d, e

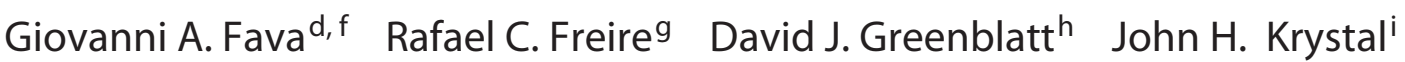 \\ Antonio E. Nardig Karl Rickels ${ }^{j}$ Thomas Roth ${ }^{k}$ Carl Salzman' Richard Shader ${ }^{\text {h }}$ \\ Edward K. Silberman $^{h}$ Nicoletta Sonino ${ }^{d, m}$ Vladan Starcevic ${ }^{n}$ \\ Steven J. Weintraub ${ }^{\circ}$

\begin{abstract}
a'Wayne State University, Detroit, MI, USA; ${ }^{\text {}}$ McGill University, Montreal, QC, Canada; ' University of Firenze,

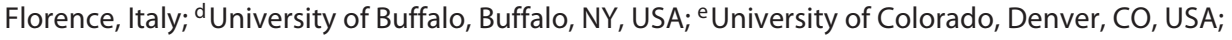
fUniversity of Bologna, Bologna, Italy; ${ }^{9}$ Federal University of Rio de Janeiro, Rio de Janeiro, Brazil; ${ }^{h}$ Tufts University, Boston, MA, USA; 'Y Yale University, New Haven, CT, USA; 'University of Pennsylvania, Philadelphia, PA, USA; ${ }^{k}$ Henry Ford Hospital, Detroit, MI, USA; 'Harvard University, Boston, MA, USA;

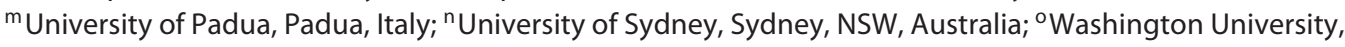
Saint Louis, MO, USA
\end{abstract}

This brief editorial is a statement to introduce a new working group on benzodiazepines, the International Task Force on Benzodiazepines, which comprises independent scientists, clinical researchers, and clinical psychopharmacologists. No references are included here as it would be beyond the scope and goal of this introduction, but a full review on benzodiazepines will be the topic of a number of papers and presentations in the near future.

Benzodiazepines have been with us since the dawn of modern psychopharmacology. Chlordiazepoxide, the first benzodiazepine, was discovered by Leo Sternbach in the late 1950s and was approved for use in the USA in 1960. Sternbach, a genial chemist, also discovered several other benzodiazepines, such as clonazepam, diazepam, flurazepam, flunitrazepam, and nitrazepam.

Benzodiazepines quickly became popular and widely used due to their versatility, tolerability, and ease of use. As they have anxiolytic, anticonvulsant, hypnotic, muscle

\section{KARGER}

() 2018 S. Karger AG, Basel

E-Mail karger@karger.com

www.karger.com/pps relaxant, and sedative properties, they have been used widely and remain the most widely prescribed psychotropic medications among all medical specialties. Psychiatrists have been using benzodiazepines for the treatment of anxiety disorders, insomnia, alcohol withdrawal, and as adjunct therapy for many other indications since their discovery. The anxiolytic properties of benzodiazepines are still unsurpassed by other psychotropic medications, such as antidepressants and antipsychotics that are used in the treatment of anxiety disorders and anxiety symptoms in other mental disorders. Their adverse effect profile is relatively benign, with sedation and possible cognitive impairment being noted most frequently.

In spite of the unquestionable benefits of benzodiazepines and their popularity among physicians of various disciplines, we have witnessed a fairly negative campaign against benzodiazepines, which are often described as being readily abused (although their abuse liability is low and, if abuse occurs, it is in the context of other substance 
abuse). Interestingly, this campaign has intensified since the advent of selective serotonin reuptake inhibitors (SSRIs) in the mid-1990s. The SSRIs, originally approved for the treatment of depressive disorders, were quickly approved for various anxiety disorders despite the lack of sufficient evidence (i.e., comparison to the existing efficacious anxiolytic drugs, benzodiazepines), and they are now promoted as the first-line treatment for these disorders. In addition, the scientific literature has gradually and surreptitiously been flooded with more and more articles on "negative" properties of benzodiazepines. While many of these publications have either not been based on good science or been frankly biased, they easily achieved a common goal that negative propaganda frequently reaches: they aroused suspicion of benzodiazepines and suggested difficulties in using them, while overlooking their benefits. An "illusion of truth" effect then occurred as frequently repeated negative information and halftruths gradually became the truth as benzodiazepines were given a "bad" name and their reputation was damaged, especially in some scientific circles. Even prescribing these drugs has become a cumbersome procedure around the world.

The International Task Force on Benzodiazepines, as a group of investigators and clinical psychopharmacologists with long-standing clinical and scientific expertise, has been concerned about this excessively negative trend. We feel that benzodiazepines have not been given proper attention during the last 2-3 decades, they have not been adequately compared to other psychotropic medications in various indications, and their risks and side effects have been overemphasized. Some of us feel that benzodiazepines have been the subject of an unspoken "commercial war."

This Task Force will be working on presenting various psychiatric and medical audiences with information about benzodiazepines which is evidence based, balanced, unbiased, and clinically relevant and useful. We believe that our colleagues deserve such information as it will encourage our common goal of treating our patients effectively, properly, and safely. We hope to preserve benzodiazepines as a valuable part of our armamentarium.

\section{Disclosure Statement}

Dr. Balon, Dr. Chouinard, Dr. Cosci, Dr. Fava, Dr. Freire, Dr. Greenblatt, Dr. Nardi, Dr. Rickels, Dr. Salzman, Dr. Shader, Dr. Silberman, Dr. Sonino, Dr. Starcevic, and Dr. Weintraub have no conflicts of interest.

Dr. Dubovsky received support from Janssen, Otsuka, Intracellular Therapies, Boehringer Ingelheim, Johnson \& Johnson, and Patrick Lee Foundation.

Dr. Krystal reports the following support. Sources of research support: Department of Veterans Affairs, VA National Center for PTSD; Department of Veterans Affairs/Department of Defense, Consortium for Alleviation of PTSD; National Center for Advancing Translational Science, NIH; National Institute on Alcohol Abuse and Alcoholism, P50 (CTNA); National Institute of Mental Health, FAST-Psychosis Consortium. Paid editorial relationship: Biological Psychiatry (editor). Scientific advisory boards: biOasis Technologies Inc., Biohaven Pharmaceuticals; Blackthorn Therapeutics Inc.; Broad Institute at MIT and Harvard; Lohocla Research Corporation; Luc Therapeutics Inc.; Pfizer Pharmaceuticals; TRImaran Pharma. Stocks: ArNETT Neuroscience Inc.; Biohaven Medical Sciences; Blackthorn Therapeutics Inc.; Spring Care Inc. Stock options: Biohaven Pharmaceuticals Medical Sciences; Luc Therapeutics Inc. Consulting relationships: AstraZeneca Pharmaceuticals; Biogen, Idec, MA; Biomedisyn Corporation; Janssen Research \& Development; L.E.K. Consulting; Otsuka America Pharmaceuticals Inc.; Pragma Therapeutics; S K Life Science; Spring Care Inc.; Sunovion Pharmaceuticals Inc.; Takeda Industries; Taisho Pharmaceuticals Co. Ltd; Teva Branded Pharmaceutical Products R\&D Inc. Patents: Dopamine and Noradrenergic Reuptake Inhibitors in Treatment of Schizophrenia; Glutamate Modulating Agents in the Treatment of Mental Disorders; Intranasal Administration of Ketamine to Treat Depression; Methods of Treating Suicidal Ideation. Provisional patents: Composition and Method to Treat Addiction; Treatment Selection for Major Depressive Disorder; Compounds Compositions and Methods for Treating or Preventing Depression and Other Diseases. Speaker's Bureau: None.

Dr. Roth has consulted for Merck, Pfizer, Novartis, Jazz, Purdue Eisai, SEQ, Avadel, GSK, Pernix, and Sanofi. 